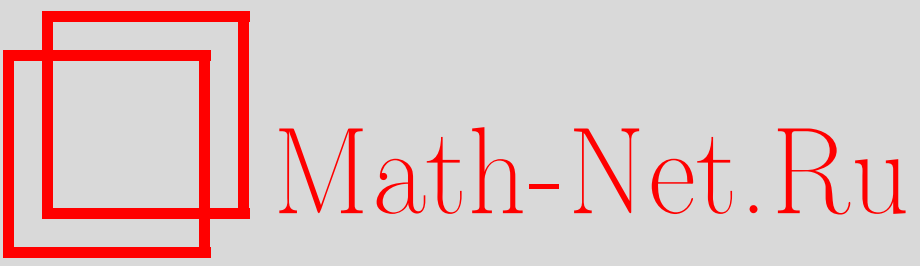

С. В. Людковский, Меры на группах диффеоморфизмов неархимедовых банаховых многообразий, УМН, 1996, том 51, выпуск 2, 169-170

DOI: https://doi.org/10.4213/rm960

Использование Общероссийского математического портала Math-Net.Ru подразумевает, что вы прочитали и согласны с пользовательским соглашением

http://www.mathnet.ru/rus/agreement

Параметры загрузки:

IP: 18.209 .158 .208

26 апреля 2023 г., 17:49:24 


\title{
МЕРЫ НА ГРУППАХ ДИФФЕОМОРФИЗМОВ НЕАРХИМЕДОВЫХ БАНАХОВЫХ МНОГООБРАЗИЙ
}

\author{
С. В. Людковский
}

В работе [1] были построены квази-инвариантные меры (КИМ) на группах диффеоморфизмов (ГД) $\operatorname{Diff}(t, M)$ классов гладкости $C(t)$ локально-компактных многообразий (ЛКМ) $M$ над $\mathbb{R} \mathrm{c}$ $t=(l, b)$ при $l \in \mathbb{N}$ и $0 \leqslant b<1$. В данной статье исследован случай банаховых метризуемых сепарабельных аналитических многообразий (БМ) $M$ над неархимедовыми ЛК полями $K \supset \mathbb{Q}_{p}$ с $T x M=X-$ банаховыми пространствами (БП) $X[2]$.

Каждая непрерывная функция $f: M \rightarrow K$ имеет единственное разложение $f(x)=$ $\sum\left\{a(m, f) Q_{m}(x): m \in \mathbb{N}_{0}^{n}\right\}$, где $Q_{m}(x)$ и $P_{m}(x)$ определены в [3], [4], а $M:=B\left(K^{n}, 0,1\right)$, $\mathbb{N}_{0}:=\mathbb{N} \cup\{0\}$. Пусть $j(b, x):=p^{z}$ с $z=b \cdot \operatorname{ord}_{p} x$ для $x \neq 0$ и $j(b, 0):=0$, для $h: M \rightarrow K:$ $\left(\Phi^{b e(i)} h\right)(y, y+t e(i)):=[h(t e(i)+y)-h(y)] / j(b, t)$ и $\Phi^{j} h$ для $j=j(i) e(1)+\cdots+j(n) e(n)$, $0 \leqslant j(i):\left(\Phi^{j} h\right):=\left(\Phi^{l}\left(\Phi^{b^{\prime}} h\right)\right)$, где $j(i):=l(i)+b(i), l(i) \in \mathbb{N}_{0}, 0 \leqslant b(i)<1$, и $b^{\prime}:=$ $\{b(1), \ldots, b(n)\},\left|b^{\prime}\right| \leqslant b$. Тогда $C(l, b, M \rightarrow K):=\left\{f: M \rightarrow K:\left(\Phi^{v} f\right)\right.$ непрерывны на $M^{s}$, $\|f\|:=\sup \left\{\left|\left(\Phi^{v} f\right)\right|:\right.$ на $\left.\left.M^{s}, s=|j|+\operatorname{sign} b(1)+\cdots+\operatorname{sign} b(n)+1,|v| \leqslant l+\operatorname{sign} b\right\}\right\}$. Пусть $X$ и $Y-$ БП над $K, F: U \rightarrow Y$, где $U$ открыто в $X$. Назовем $F$ дифференцируемым, если $\forall t$ в $K, x$ в $U$ и $h$ в $X$ с $x+t h$ в $U: D F(x, h)=F^{\prime}(x) h$, где $F^{\prime}(x)$ - линейный непрерывный оператор (по $h$ ) и $D F(x, h):=\{d F(x+t h) / d t \mid\{t=0\}\}:=\lim _{t \rightarrow 0}\{F(x+t h)-F(x)\} / t$, $\Phi^{1} F(x, h, t):=\{F(x+t h)-F(x)\} / t$, имеет непрерьвное продолжение на $U \times V \times S, U$ и $V$ - ОЗ окрестности $x$ и $0 \in X, S=B(K, 0,1)$, а $\left\|\Phi^{1} F(x, h, t)\right\|:=\sup \left\{\left\|\Phi^{1} F(x, h, t)\right\| /\|h\|\right.$ : $x \in U, h \in V, t \in S\}<\infty$ и $\Phi^{1} F(x, h, 0)=F^{\prime}(x) h$. По индукции и с использованием $j(b, t)$ определяются $\Phi^{n, j} F(x, h(1), \ldots, h(n+1) ; t(1), \ldots, t(n+1))$ и БП $C(l, b, U \rightarrow Y)$.

ГД $\operatorname{Diff}(t, M)$ несепарабельны и их число Суслина несчетно, если $\operatorname{dim}_{k} M=\infty$, поэтому далее через $G(t)$ обозначены сепарабельные подгруппы: для $f$ из $G(t)$ имеется разложение $f(x)=\sum\left\{a(m, f(i, *)) Q_{m}(x) e(i): i, n \in \mathbb{N}, m \in \mathbb{N}_{0}^{n}, x \in K^{n}, \lim \{\mid a(m, f(i, *)-\right.$ $\operatorname{id}(i, *))|J(t, m):| m \mid+n+i \rightarrow \infty\}\}=0$ на $B(X, 0,1)$, где $J(t, m):=\left\|Q_{m}\right\|_{C(t)}, t=$ $(l, b)$ или $t=(a n, r)$ (аналитических на $B(X, 0, r)$ ). Топология на $G(t)$ дается семейством псевдометрик $d(t, V ; h, g):=\left\|g^{-1} \circ h-\mathrm{id}\right\|, \mathrm{c}\|h\|:=\sup \{\mid a(m, h(i, *) \mid J(t, m): i, n, m\}$, где $V$ гомеоморфно $B(X, 0,1)$ и $c=(V, f, X)$ - карта атласа $\operatorname{At}(M)$ на $M$. Каждая $f$ из БП $C(t, \inf , B(X, 0,1) \rightarrow X)$ имеет разложение $f(x)=\sum\{f(i, n ; x) e(i) z(n): i \in \mathbb{N}$, $\left.n \in \mathbb{N}_{0}\right\},\left\{e(i) z(n) Q_{m}(x): i, n, \operatorname{Ord} m:=\max (i: m(i) \neq 0)=n\right\}$ - ортогональный базис (ОБ) [5], $f(x ; n):=\sum\{f(i, n ; x) e(i): i\} \in C\left(t, B\left(K^{n}, 0,1\right) \rightarrow X\right)$, где $f(i, n ; x):=$ $\left\{a(m, f(i, *)) Q_{m}(x): \operatorname{Ord} m=n, m=(m(1), \ldots, m(n)), m(j) \in \mathbb{N}_{0}\right\}$. В некоторой окрестности $G(t) \supset W^{\prime \prime} \ni$ id плотно семейство элементов, принадлежащих однопараметрическим подгруппам $\left\{g^{v}: v \in \mathbb{Z}_{p}\right\}(\mathrm{OП})$. Пусть $S(v, f)=g(v, f)$ - ОП, задаваемая потоком $S$ с $d S(v, f) / d v=A(x) S(v, f(x)), S(v+z, f)=S(v, S(z, f))$ и $S(v, m)(B):=m(S(-v, B))$, тогда $m$ называется псевдодифференцируемой вдоль векторного поля класса $C\left(t^{\prime \prime}\right), A(x) \in$ $\operatorname{Vect}\left(t^{\prime \prime}, M\right)$, если существует $\operatorname{PD}(b, S(v, m)(B))$ по $v \forall B \in \operatorname{Bf}(G(t))$, где $\operatorname{PD}(b, h(v)):=$ $\int_{\mathbb{Z}_{p}}[h(v)-h(s)] z(b, v-s) d w(s), z(b, v):=q^{a}, a=-(1+b) \cdot \operatorname{ord}_{p} v$ или $z(b, v):=|v-s|^{a}$, $a=-1-b$ для $m$ и меры Хаара $(\mathrm{MX}) w$ со значениями в $\mathbb{Q}_{q}$ и $\mathbb{R}$, соответственно.

ТЕОремА 1. ГД $G(t)$ имеет КИМ $m$ относительно плотной подгруппь $G^{\prime}$, u $m$ псевдодифференцируема $\forall b \in \mathbb{C}$ относительно $S(v, *)$ из плотной в $W^{\prime \prime}$ подгруппь $G^{\prime \prime}$.

Для доказательства рассмотрим $\{s(n) \in \mathbb{N}: n \in \mathbb{N}\}$, такие что $s(n)<s(n+1) \forall n$, $A(f):=\sum\left\{q(k) A\left(k ; f^{\prime}, P(n) f\right) z(n): n \in \mathbb{N}, k=(k(1), \ldots, k(n)), k(j) \in \mathbb{N}_{0}, n \in \mathbb{N}\right\}$, $A\left(k ; f^{\prime}, P(n) f\right):=\partial_{1}^{2 i(1)} \ldots \partial_{r-1}^{2 i(r-1)} \partial_{r+1}^{2 i(r+1)} \ldots \partial_{n}^{2 i(n)} \sum\left\{y(l) \partial_{r}^{l}\left[\left(f^{\prime}\right)^{-1} \partial_{r}^{2 i(r-l)} f(x ; n) \quad:\right.\right.$ $l=0, \ldots, s-1\}$ c $|k|=s(n) n, \sum\{q(k): \operatorname{Ord} k \leqslant n,|k|=s(n) n\}=: t(n), 0<c \leqslant$ $|t(n)| \leqslant c^{\prime}<\infty \forall n$ с фиксированнтми $c$ и $c^{\prime}$, где $\{y(l)\}$ - решения соответствующей системы линейных алгебраических уравнений [1], $P(n)$ - проекционный оператор на 
$X_{n}=\{f(x ; n): f \in C(t)\}, P(n) f(x)=f(x ; n)$, где $\{e(i): i \in \mathbb{N}\}-$ нормированный ОБ (ОНБ) в $X, M=B(X, 0,1)$. Можно задать $R(f):=\sum\{q(k) R(k, n ; f) z(n): n \in \mathbb{N}$, $k=(k(1), \ldots, k(n))\}$, где $L=T^{-1}, T(l, b): C(l, b, M) \rightarrow C(a n, M)$ - линейный топологический изоморфизм $R(k, n ; f):=\partial^{a u} F(k, n ; Q(f ; n)), F(k, n ; f):=L[A]\left(k ; g^{\prime}, P(n) g\right), g=T f$, $Q(f ; n):=Q(l+a, Q(l+a-1, \ldots, Q(l+1, f(x ; n)) \ldots), l+a \geqslant 1$, здесь каждый $Q(l+j, *)$ действует по $(x(1), \ldots, x(n)), L$ действует на $([A] T(l, b) f)(y+a+b+\cdots+z, y+b+\cdots+z, \ldots, y+z, y)$ по $y$, так что $L([A] T(l, b) f)(y, \ldots, y)=L A T(l, b) f(y)$, оператор $[A]$ обозначает $A$ с заменой всех $\partial_{i}$ на $\Phi^{e(i)}$. Пусть $f(x) \in C\left(l-1, b, B\left(K^{n}, 0,1\right) \rightarrow K\right)$, тогда антидифференцирование $P(l)$ зададим формулой: $P(l) f(x):=\sum\left\{\partial^{j} f\left(x_{m}\right)\left(x_{m+u}-x_{m}\right)^{j+u} /(j+u) !: m \in \mathbb{N}_{0}^{n}\right.$, $|j|=0, \ldots, l-1\}$, где $u=(1, \ldots, 1) \in \mathbb{N}^{n}, x_{m}=\sigma_{m}(x),\left\{\sigma_{m}: m \in \mathbb{N}_{0}^{n}\right\}-$ приближение 1 в $B\left(K^{n}, 0,1\right)[4]$, a $Q(l, f):=P(l)(f-\mathrm{id})+\mathrm{id}$.

Пусть $\|[g](y)\|_{E(a n,-k)}=\sup \{|a(j, f)| J(a n, j) / J(k, 0, j): j\}$, где $E(a n, 0, N)=C(a n, N)$, $[g](y)=[A] f, y \in N$, т.е. $[A] C(a n, M)=E(a n,-k, N)$ при $M=B\left(K^{n}, 0,1\right)$, так как $[\Delta]:$ $C(a n, M, 0) \rightarrow E\left(a n,-2, M^{3} \rightarrow K\right)$ - линейный топологический изоморфизм, разностное уравнение для $[\Delta]$ можно решить по индукции, взять соответствующую карту и воспользоваться теоремой об обратной функции. Здесь для $f(V)=B\left(K^{n}, 0,1\right)=: S$ по определению $\partial S=$ $[x=(x(1), \ldots, x(n)): \exists j$ с $x(j)=0$ или 1], $\partial M:=U\{\partial f(V): c \in$ At $:=(c=(V, f, K))\}$. Аналогично в бесконечномерном случае и $C(t, M, h):=\{f \in C(t, M): f \mid\{\partial M\}=0$ при $j=0, \ldots, v(h, n)\}$ с $v(h, n)=2 h-1$ при четном $l$ и $2 h$ при нечетном $l, G(t, h):=\{f \in$ $G(t):\{(f(u ; x)-\operatorname{id}(u ; x)): u=0, \ldots, n\} \in C(t, M, h(n)), h(n)=[s(n) n / 2], n \in \mathbb{N}\}$, $h:=\{h(n): n \in \mathbb{N}\}$, где $s(n)=n+1$. Для неограниченного $M$ "граничные" условия задаются на каждой $\partial U(k)$, т.е. подмногообразии, регулярно вложенном в $M$ и коразмерности 1 над $K$.

Пусть $\{H(n):=E(t,-2 s(n) n, N \rightarrow X) \mid n \in \mathbb{N}\}$ - последовательность БП над $K$, а $c_{0}(H(n): n \in \mathbb{N}):=\left\{f=(f(1), f(2), \ldots): f(n) \in H(n) \forall n\right.$ и $\left.\lim _{n \rightarrow \infty}\|f(n)\|_{H(n)}=0\right\}$ $=: E$, где $t=(l, b)$ или $t=(a n, r), N=M^{v+1}, v=2 s(n) n$. Тогда при $M=B(X, 0,1)$ существует ОЗ подгруппа $W$ в $G(t, h)$ локально гомеоморфная ОЗ окрестности 0 в банаховом пространстве $Y$ в $E$. Использя (а) произведения мер $v(d x)=h(x) w(d x)$, задаваемых с помощью $\mathrm{MX} w$ на $\mathbb{Q}_{p}$ с подходящими локально-постоянными $h$ на $\mathbb{Q}_{p}$ со значениями в $\mathbb{R}$ или $\mathbb{Q}_{q}(q \neq p),(б)$ теорему Прохорова [6], (в) модифицируя теоремы из $\S 26$ в [7], получим КИМ $m$ на $G(t)$.

TeOpema 2. Пусть $G=G(a n, r) u \operatorname{dim}_{K} M=n \in \mathbb{N} \cup\{\infty\}$, mогда существует O3 подгруппа $W$, такая что каждая $g \in W$ принадлежит соответствующей ОП, но функция $\exp : \operatorname{Vect}(a n, M) \rightarrow G$ не является локально биективной.

Рассмотрим $W:=\left\{f \in G:\|f-\mathrm{id}\| \leqslant p^{-2}\right\}$ и $g(j ; x):=\sum\left\{A(j ; x)^{s} x / s !: s=0,1, \ldots\right\}$, используя разложения по $Q_{m} e(i)$, достаточно рассмотреть полиномы и $1 \leqslant i \leqslant n \in \mathbb{N},|m| \leqslant k$, где $A(j ; x):=\sum\left\{T(j, i ; x) \partial_{i}: i=1, \ldots, n\right\}, T(j, i ; x)$ - полиномы на $j$-м шаге, $P(i ; x):=$ $T(0, i ; x), P(x):=(f-\mathrm{id})(x)=\sum\{P(i ; x) e(i): i=1, \ldots, n\}, A(0 ; x) A(1 ; x)+A(1 ; x)=P^{\wedge}(x)$, $P^{\wedge}(x):=\sum\left\{P(i ; x) \partial_{i}: i\right\}, P^{\wedge}(x)=A(j ; x)+\sum\left\{A(j-1 ; x)^{s} A(j ; x) / s !: s=1, \ldots, j\right\}$ при $j>1$. Тогда существует $\lim _{j \rightarrow \infty} A(j ; x)=: A(j) \in \operatorname{Vect}(a n, r)$ с $W \supset \exp \{v A(x)\} x=g(v, x)$ при $v \in \mathbb{Z}_{p}, g(1, x)=f(x)$.

\section{СПИСОК ЛИТЕРАТУРЫ}

[1] Шавгулидзе Е. Т. // ДАН СССР. 1988. Т. 303. С. 811-814. [2] Бурбаки Н. Дифференцируемые и аналитические многообразия. М.: Мир, 1975. [3] Amice Y.// Bull. Soc. Math. France. 1964. V. 92. P. 117-180. [4] Schikhof W. H. Ultrametric calculus. Cambridge: Cambridge Univ. Press, 1984. [5] van Rooij A. C. M. Non-Archimedean functional analysis. New York: Marcel Dekker, 1978. [6] Бурбаки Н. Интегрирование. Гл. 1-9. М.: Наука, 1970, 1977. [7] Скороход А. В. Интегрирование в гильбертовом пространстве. М.: Наука, 1975. 